\title{
Interference of Single Photons Emitted by Entangled Atoms in Free Space
}

\author{
G. Araneda, ${ }^{1, *}$ D. B. Higginbottom, ${ }^{1,2}$ L. Slodička, ${ }^{3}$ Y. Colombe, ${ }^{1, \dagger}$ and R. Blatt ${ }^{1,4}$ \\ ${ }^{1}$ Institut für Experimentalphysik, Universität Innsbruck, Technikerstraße 25, 6020 Innsbruck, Austria \\ ${ }^{2}$ Centre for Quantum Computation and Communication Technology, Research School of Physics and Engineering, \\ The Australian National University, Canberra ACT 2601, Australia \\ ${ }^{3}$ Department of Optics Palacký University, 17. Listopadu 12, 77146 Olomouc, Czech Republic \\ ${ }^{4}$ Institut für Quantenoptik und Quanteninformation, Österreichische Akademie der Wissenschaften, \\ Technikerstraße 21a, 6020 Innsbruck, Austria
}

(Received 30 November 2017; published 11 May 2018)

\begin{abstract}
The generation and manipulation of entanglement between isolated particles has precipitated rapid progress in quantum information processing. Entanglement is also known to play an essential role in the optical properties of atomic ensembles, but fundamental effects in the controlled emission and absorption from small, well-defined numbers of entangled emitters in free space have remained unobserved. Here we present the control of the emission rate of a single photon from a pair of distant, entangled atoms into a freespace optical mode. Changing the length of the optical path connecting the atoms modulates the singlephoton emission rate in the selected mode with a visibility $V=0.27 \pm 0.03$ determined by the degree of entanglement shared between the atoms, corresponding directly to the concurrence $\mathcal{C}_{\rho}=0.31 \pm 0.10$ of the prepared state. This scheme, together with population measurements, provides a fully optical determination of the amount of entanglement. Furthermore, large sensitivity of the interference phase evolution points to applications of the presented scheme in high-precision gradient sensing.
\end{abstract}

DOI: 10.1103/PhysRevLett.120.193603

Collective emission and absorption properties of entangled emitters have been extensively studied in ensembles of neutral atoms [1-4], where, in general, the number of emitters fluctuates and the control of the quantum state of each emitter is challenging. To observe the coherent interaction of light with a definite number of entangled emitters, it is necessary to achieve simultaneously subwavelength emitter localization, high-fidelity entanglement generation, and atom-light coupling strong enough to detect an optical signal at very low photon flux. Experimental systems motivated by quantum computation have enabled excellent control over position and entanglement with well-defined and steadily increasing numbers of particles [5-7]. Meanwhile, the pursuit of on-demand single photons, strong atom-light coupling, and fast quantum state readout has advanced the collection efficiency of light from single emitters [8-11]. Together these developments enable the investigation of entanglement in collective atom-light interactions in the few-atom limit $[12,13]$.

Previous experiments have shown free-space or cavitymediated interference from atoms in separable states [14-16], where each atom emits a photon independently, or demonstrated enhancement and suppression of singlephoton emission in a cavity mode from an entangled state of two atoms [13]. In this Letter we present the first observation of interference in the spontaneous emission of a single photon into free space, emitted jointly by a pair of atoms prepared in a well-characterized entangled state.
The atoms are trapped adjacently but share a common optical mode in which their effective optical separation is $d \simeq 60 \mathrm{~cm}$. The interference we observe in the singlephoton emission probability arises solely from the entanglement present in the two-atom state [17]. With this arrangement we directly observe both enhancement and inhibition of single-photon emission from the atoms by controlling the optical distance between them in the common mode. This distance can be set dynamically during an experimental sequence, with subwavelength precision and over timescales well below the lifetime of the entangled state.

In order to study the optical properties of entangled particles, we first entangle two atoms. Entanglement can be achieved, e.g., with schemes relying on motional and internal atomic states [18-20], or through the coincident detection of two photons [16]. Here we use a scheme requiring only the detection of a single photon, proposed by Cabrillo et al. [21] and demonstrated in Ref. [22]. Two Doppler-cooled ${ }^{138} \mathrm{Ba}^{+}$ions, with electronic structure shown in Fig. 1(a), initially prepared in an electronic ground state $\left|g_{-}\right\rangle=\left|6 S_{1 / 2}, m_{j}=-\frac{1}{2}\right\rangle$ are excited to the state $\left|g_{+}\right\rangle=\left|6 S_{1 / 2}, m_{j}=+\frac{1}{2}\right\rangle$ with single-atom transition probability $p_{e}=6 \pm 1 \%$ through the spontaneous Raman process $\left|g_{-}\right\rangle \rightarrow|i\rangle \rightarrow\left|g_{+}\right\rangle$by weak laser excitation to the intermediate, short-lived excited state $|i\rangle=\mid 6 P_{1 / 2}$, $\left.m_{j}=+\frac{1}{2}\right\rangle$; see Fig. 1(b). A magnetic field $\vec{B}$ is applied 
(a)

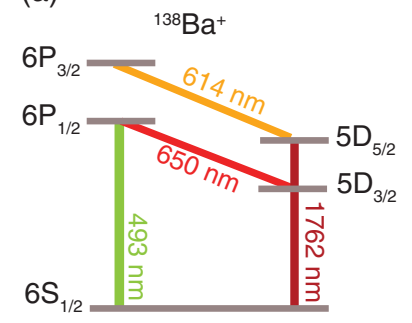

(b)

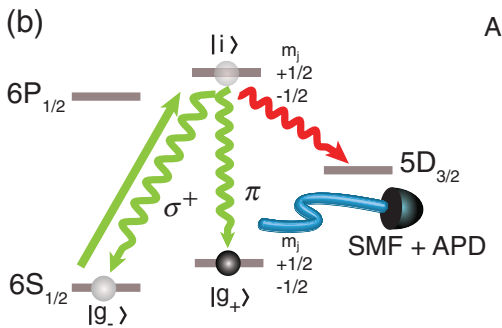

(c)

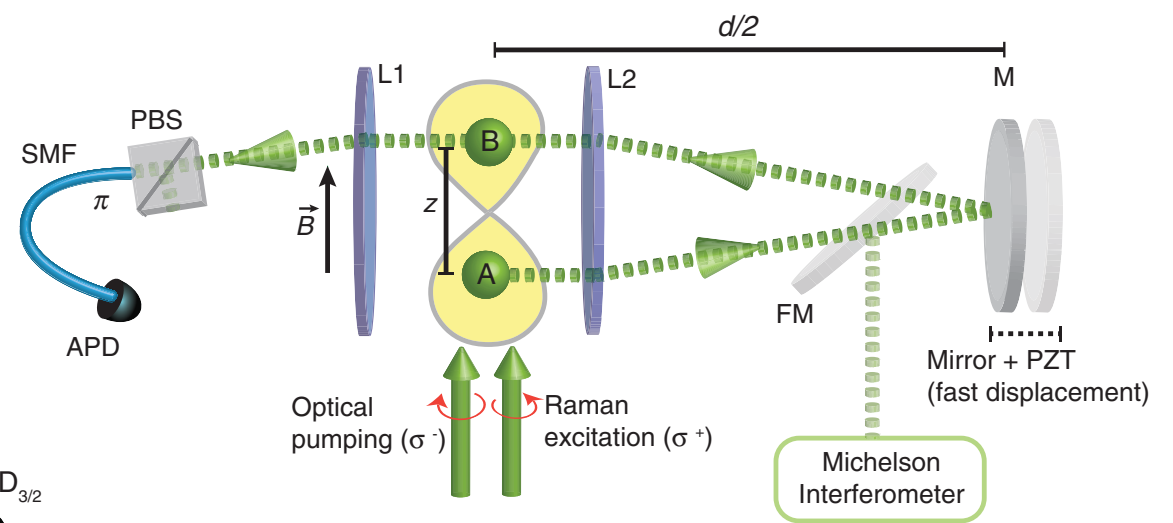

FIG. 1. (a) Electronic levels of ${ }^{138} \mathrm{Ba}^{+}$. (b) Excitation of the short-lived intermediate state $|i\rangle$ by a $\sigma^{+}$beam at $493 \mathrm{~nm}$. Detecting a $\pi$ polarized herald photon during a weak excitation pulse prepares a two-atom entangled state. A further, stronger excitation scatters a $\pi$ polarized witness photon from the entangled state in the common mode with a probability that depends on the phase difference $\Delta \phi$. (c) Two ${ }^{138} \mathrm{Ba}^{+}$ions, $A$ and $B$, are trapped and cooled in a linear Paul trap, separated by a distance $z \simeq 5.2 \mu \mathrm{m}$. The radiation fields are collimated using two identical in-vacuum high numerical aperture lenses ( $L 1$ and $L 2)$. A mirror $(M)$ at a distance $d / 2 \simeq 30 \mathrm{~cm}$ superimposes emissions from the ions so that they are coupled to a common spatial mode. The mirror is mounted on piezotransducers (PZT) that allow fast subwavelength control of the atom-atom distance in the common optical mode. A polarizing beam splitter (PBS) selects $\pi$-polarized photons, which are coupled to a single-mode fiber (SMF) and detected by an avalanche photodiode (APD). A Michelson interferometer enabled by a flip mirror (FM) is used to calibrate the PZT-mirror voltage-displacement response.

along the trap axis, defining the quantization axis. Part of the emission of the atoms is coupled to a $\pi$-polarized, wavelength $\lambda=493 \mathrm{~nm}$ common mode by using invacuum lenses ( $L 1$ and $L 2)$ and the distant mirror $(M)$; see Fig. 1(c). The emission is coupled into a single-mode fiber defining the spatial mode, together with a polarizing beam splitter defining its polarization. Detection of a single "herald" photon in this common mode projects the atoms onto the entangled state [22],

$$
|\psi\rangle=\frac{1}{\sqrt{2}}\left(\left|g_{+}, g_{-}\right\rangle+e^{i \phi}\left|g_{-}, g_{+}\right\rangle\right)
$$

which belongs to a decoherence-free subspace [23]. The probability of generating this state is $2 p_{e}\left(1-p_{e}\right) \eta$, where $\eta$ is the overall detection efficiency of a single photon. The phase $\phi$ of the entangled state is given by

$$
\phi=\left(\phi_{L_{B}}-\phi_{L_{A}}\right)+\left(\phi_{D_{B}}-\phi_{D_{A}}\right)
$$

where $\phi_{L_{B}}-\phi_{L_{A}}=k z$ is the phase difference of the exciting laser field at the positions of the atoms $A$ and $B, \phi_{D_{B}}-\phi_{D_{A}}=-k d$ is the phase difference associated with the path followed by a photon emitted by atom $A$ or $B$ on its way to the detector, and $k=2 \pi / \lambda$.

We estimate a fidelity $F=0.65 \pm 0.02$ between the experimentally generated state and the ideal entangled state $|\psi\rangle$ by measuring parity oscillations [24]. The spatial, spectral, and polarization indistinguishability of the detected photons is characterized by the value of the second-order correlation function at zero delay $g^{(2)}(t=0)=$ $0.99 \pm 0.06$, close to perfect indistinguishability, so that the fidelity is limited by other factors, primarily atomic motion (for details on entanglement generation and characterization, see Supplemental Material, Secs. I and II [25]). The herald photon detection rate, which corresponds to the rate of entanglement generation, is $5.02 \mathrm{~s}^{-1}$.

If we further excite the entangled atom pair with the $\sigma^{+}$-polarized beam, only the component of the entangled state in $\left|g_{-}\right\rangle$may absorb a photon and spontaneously decay to $\left|g_{+}\right\rangle$, emitting a second photon. The state of the joint atom-photon system after completing this second Raman process is

$$
\left|\psi^{\prime}\right\rangle=\frac{1}{\sqrt{2}}\left(|0,1\rangle+e^{i\left(\phi_{L_{A}}-\phi_{L_{B}}\right)} e^{i \phi}|1,0\rangle\right)\left|g_{+}, g_{+}\right\rangle .
$$

The field states $|1,0\rangle$ and $|0,1\rangle$ correspond to the emission of a single photon from atom $A$ or $B$. The detection of a "witness" photon in the common mode projects the atoms onto the unnormalized state,

$$
\left|\psi_{p}^{\prime}\right\rangle=\frac{1}{\sqrt{2}}\left(1+e^{i\left(\phi_{L_{A}}-\phi_{L_{B}}+\phi_{D_{A}}^{\prime}-\phi_{D_{B}}^{\prime}\right)} e^{i \phi}\right)\left|g_{+}, g_{+}\right\rangle,
$$

where $\phi_{D_{A}}^{\prime}-\phi_{D_{B}}^{\prime}=k d^{\prime}$ is the optical phase difference in the common mode when the witness photon is 


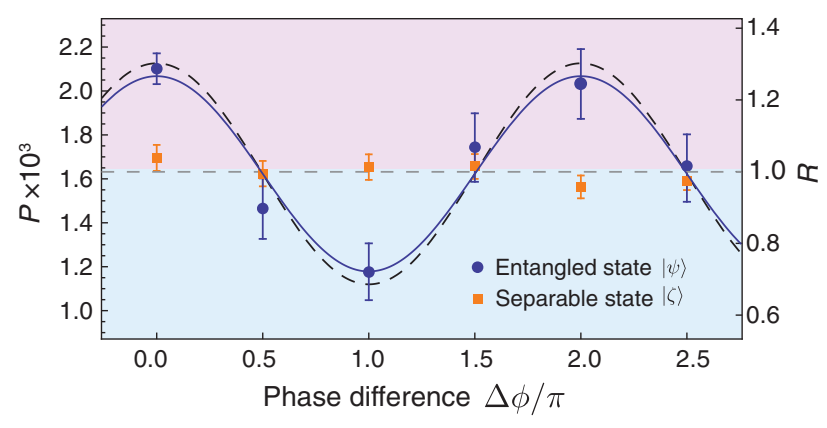

FIG. 2. Absolute $(P)$ and relative $(R)$ witness photon probability from the atom pair as a function of the phase difference $\Delta \phi$ for the entangled state $|\psi\rangle$ (blue circles) and the separable state $|\zeta\rangle$ (orange squares). Error bars correspond to the Poissonian error from photon counting. The blue solid curve is the amplitude and offset fitted scattering model for the entangled state, while the black dashed curve shows the expected probability from the independent estimation of its concurrence. The gray dashed line is the average of the fitted curve. The witness photon detection probability for $|\psi\rangle$ is maximally enhanced at $\Delta \phi=0,2 \pi$ and maximally suppressed at $\Delta \phi=\pi$. The photon detection probability for $|\zeta\rangle$ is constant within the measurement uncertainty.

detected. The probability of detecting a single witness photon is

$$
P \propto\left|\left\langle\psi_{p}^{\prime} \mid \psi_{p}^{\prime}\right\rangle\right|^{2}=1+\cos \left(\phi-\phi^{\prime}\right),
$$

where $\phi^{\prime}=\left(\phi_{L_{B}}-\phi_{L_{A}}\right)+\left(\phi_{D_{B}}^{\prime}-\phi_{D_{A}}^{\prime}\right)$. The witness photon detection probability is modulated by the phase difference $\Delta \phi=\phi-\phi^{\prime}$ between the herald and witness detection events. This effect is a consequence of entanglement between the two emitters and corresponds to enhancement or inhibition of the emission probability in the common mode due to single-photon path interference [17].

We vary the phase difference $\Delta \phi$ by rapidly changing the position of the distant mirror from $d / 2$ to $d^{\prime} / 2$ between the two detection events within a time $\tau \leq 220 \mu \mathrm{s}$, while maintaining a fixed interatomic distance $z$, so that $\Delta \phi=$ $k\left(d^{\prime}-d\right)$ (Supplemental Material, Sec. III [25]). This allows precise phase control in short times without motional excitation of the ions. In order to efficiently generate a witness photon from the entangled state, a laser pulse, stronger than the pulse used to create entanglement, is used, with a $\left|g_{-}\right\rangle \rightarrow\left|g_{+}\right\rangle$transition probability $p_{w}=$ $80 \pm 2 \%$ for a single atom. The full sequence is described in Supplemental Material, Sec. IV [25]. We measure the witness photon detection probability $P$ by counting the number of witness photons per herald photon for a given phase change $\Delta \phi$. The results of the measurement, together with the amplitude- and offset-fitted model from Eq. (5), are plotted in Fig. 2. The maximum and minimum measured probabilities are $P(\Delta \phi=0)=(2.10 \pm 0.07) \times$ $10^{-3}$ and $P(\Delta \phi=\pi)=(1.17 \pm 0.12) \times 10^{-3}$, respectively. It has been established theoretically, but not previously observed, that the visibility of the interference fringes in radiation from a pair of two-level emitters with a single excitation should be equal to the concurrence of the bipartite quantum state [26,27]; see Supplemental Material, Sec. V [25]. The visibility $V$ obtained by the fitted model implies then a concurrence of the entangled atom pair $\mathcal{C}_{\text {wit }}=V=0.27 \pm 0.03$, in agreement with the concurrence of the bipartite density matrix inferred from parity measurements $\mathcal{C}\left(\rho_{\text {par }}\right)=0.31 \pm 0.10$. The fidelity of the entangled state, and therefore the visibility of the fringes, is limited by the motion of the atoms (Supplemental Material, Sec. II [25]).

For comparison, we measure the photon detection rate for a separable two-atom state $|\zeta\rangle=\left|g_{+}, g_{-}\right\rangle$. This state is prepared by optical pumping both atoms in the $\left|g_{-}\right\rangle$state, followed by a $1.76 \mu \mathrm{m}$ addressed shelving pulse on the $\left|g_{-}\right\rangle \leftrightarrow\left|5 D_{5 / 2}, m_{j}=-\frac{5}{2}\right\rangle$ transition on atom $B$ succeeded by a global rf $\pi$-pulse between the $\left|g_{-}\right\rangle$and $\left|g_{+}\right\rangle$states of both ions, and finally unshelving the atom $B$. We trigger the emission of a witness photon from this state by using an excitation beam with the same parameters used for emitting a photon from the state $|\psi\rangle$. The witness photon detection probability for $|\zeta\rangle$, which is the mean number of photons detected per prepared state, is shown in Fig. 2 (orange squares) as a function of the optical phase $\Delta \phi$. In contrast to the entangled state $|\psi\rangle$, the photon probability $P_{|\zeta\rangle}$ for the separable state $|\zeta\rangle$ is independent of the phase difference, with an average detection probability $P_{\text {sep }}=(1.63 \pm 0.05) \times 10^{-3}$. The observed visibility for this state is $\approx 0$, in agreement with the expected vanishing concurrence of the state. We define the relative probabilities $R=P(\Delta \phi) / P_{\text {sep }}$, so that $R>1(R<1)$ represents enhanced (suppressed) detection probability relative to this separable state. The relative scale is shown in Fig. 2, right vertical axis. Because states $|\psi\rangle$ and $|\zeta\rangle$ both contain a single atom in $\left|g_{-}\right\rangle$, we expect the mean detection probabilities from each state to be equal (Supplemental Material, Sec. V [25]). The mean of the fitted interference curve for $R_{|\psi\rangle}$ in Fig. 2 is $0.99 \pm 0.08$, in close agreement with the mean of $R_{|\zeta\rangle}$. The case of a more general separable state is studied in Supplemental Material, Sec. V [25].

The witness photon detection probability $P$ is also sensitive to any phase accumulated by the entangled state between the emission of herald and witness photons. For example, the presence of a static magnetic field gradient between the ions induces a linear increase of the entangled state phase $\phi[28,29]$. We measure the magnetic field gradient due to an external permanent magnet by recording the evolution of the witness photon emission probability with a variable delay between the herald and witness photons. The results are shown in Fig. 3 for mirror displacements corresponding to $\Delta \phi=0$ (blue) and $\Delta \phi=$ $\pi / 2$ (orange). Oscillations observed in both signals correspond to a linear evolution of the entanglement phase with a $\pi / 2$ phase shift with respect to each other. The period of the 


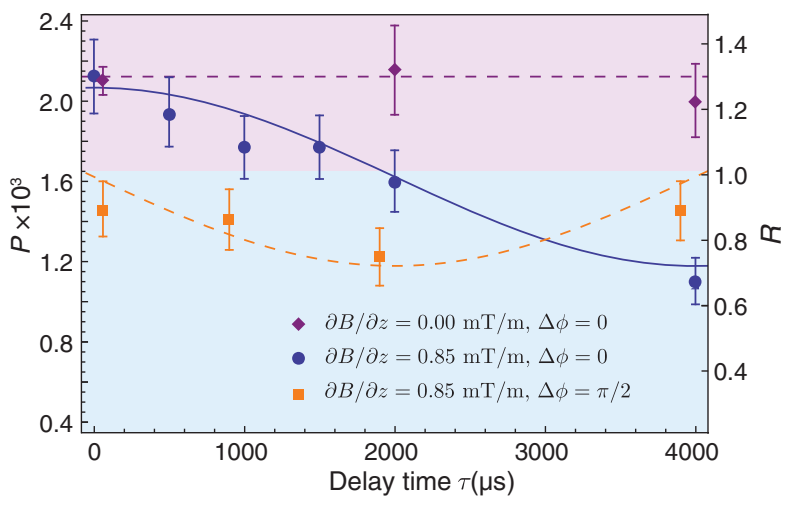

FIG. 3. Absolute $(P)$ and relative $(R)$ witness photon probability when $\Delta \phi=0$ (blue circles) and $\Delta \phi=\pi / 2$ (orange squares) for delay times $\tau$ in the presence of a magnetic field gradient compared to the same measure in the absence of a magnetic field gradient (purple diamonds) with $\Delta \phi=0$. The blue curve is amplitude and period fitted to the measured data for $\Delta \phi=0$ (blue points), while the orange dashed curve is a $\pi / 2$ phase shifted version of this fit, showing with the $\Delta \phi=\pi / 2$ measured data. The dashed purple line shows constant enhancement in the absence of magnetic field gradient.

oscillation obtained from the fit (blue curve) of the data for $\Delta \phi=0$ is $8.0 \pm 0.5 \mathrm{~ms}$. The orange curve is the same fit shifted by $\pi / 2$, and shows agreement with the measured data for $\Delta \phi=\pi / 2$. The period of the oscillation implies a magnetic field gradient of $0.85 \pm 0.05 \mathrm{mT} / \mathrm{m}$ along the ion crystal. The same measurement is taken in the absence of magnetic field gradient for $\Delta \phi=0$ (Fig. 3, purple diamonds), where the signal remains constant since dephasing effects are negligible on the measured timescale. The coherence time of the entangled state in the decoherence-free subspace is expected to be orders of magnitude longer than the timescale of the shown phase evolution [23].

While super- and subradiance, i.e., modifications of the global spontaneous emission rate, have been previously observed with a pair of trapped atoms [30], here we have shown the control of the single-photon emission rate into a selected free-space optical mode. This opens the way to experimental studies of optical properties of entangled states of a few well-controlled emitters [17,26,31,32], such as trapped ions as presented here or neutral atoms in more versatile trapping configurations [33]. The free-space configuration imposes no fundamental constraints on the direction of emission, the number of entangled particles, or their mutual distance. The phases of the emitters in the detected optical mode can be tuned arbitrarily, allowing the observation of the complete interference signal; this is in contrast to Fabry-Perot cavity systems $[12,13]$ in which the relative phase of the emitters is restricted to 0 or $\pi$ by the cavity mode. The agreement between measurements of entanglement using single-photon interference visibility and independent measurements of internal states populations confirms that the observed interference can be employed for the estimation of entanglement between distant particles, without any requirement on coherent control over internal atomic states [26,31,34]. Such a scheme is also applicable to quantum objects with different internal structures and emission spectra [35], and should allow the observation of entanglement between nonidentical atoms or general disparate quantum emitters $[5,31,36,37]$.

The spatial differences of various environmental factors are mapped directly into interference of photons from entangled particles through the evolution of the phase of the entangled state, as shown here for the case of magnetic field gradients. This points to potential applications in quantum metrology [38]; together with all-optical preparation of distant entangled states $[16,22]$ and recent developments in the stabilization of large fiber networks [39], the technique presented here may enable the development of ultrasensitive optical gradiometers [40].

We acknowledge the technical support of Muir Kumph, Matthias Brandl, Nicolas Chauvet, Bernardo Casabone, and Bryan Luu. This work has been financially supported by the Austrian Science Fund (FWF) through projects P23022 (SINPHONIA) and F4001 (SFB FoQuS), by the European Research Council (ERC) project CRYTERION No. 227959, and the Institut für Quanteninformation $\mathrm{GmbH}$. L. S. acknowledges financial support from the Grant Agency of the Czech Republic, Grant No. GB1436681G.D. B. H. acknowledges support from the Australian Research Council (ARC) (CE1101027).

*gabriel.araneda-machuca@uibk.ac.at yves.colombe@uibk.ac.at

[1] R. Schmied, J.-D. Bancal, B. Allard, M. Fadel, V. Scarani, P. Treutlein, and N. Sangouard, Science 352, 441 (2016).

[2] R. McConnell, H. Zhang, J. Hu, S. Ćuk, and V. Vuletić, Nature (London) 519, 439 (2015).

[3] M. O. Scully and A. A. Svidzinsky, Science 325, 1510 (2009).

[4] F. Haas, J. Volz, R. Gehr, J. Reichel, and J. Estève, Science 344, 180 (2014).

[5] T. R. Tan, J. P. Gaebler, Y. Lin, Y. Wan, R. Bowler, D. Leibfried, and D. J. Wineland, Nature (London) 528, 380 (2015).

[6] C. J. Ballance, V. M. Schäfer, J. P. Home, D. J. Szwer, S. C. Webster, D. T. C. Allcock, N. M. Linke, T. P. Harty, D. P. L. Aude Craik, D. N. Stacey, A. M. Steane, and D. M. Lucas, Nature (London) 528, 384 (2015).

[7] T. Monz, P. Schindler, J. T. Barreiro, M. Chwalla, D. Nigg, W. A. Coish, M. Harlander, W. Hänsel, M. Hennrich, and R. Blatt, Phys. Rev. Lett. 106, 130506 (2011).

[8] R. Maiwald, A. Golla, M. Fischer, M. Bader, S. Heugel, B. Chalopin, M. Sondermann, and G. Leuchs, Phys. Rev. A 86, 043431 (2012).

[9] E. W. Streed, A. Jechow, B. G. Norton, and D. Kielpinski, Nat. Commun. 3, 933 (2012). 
[10] G. Shu, C.-K. Chou, N. Kurz, M. R. Dietrich, and B. B. Blinov, J. Opt. Soc. Am. B 28, 2865 (2011).

[11] P. Lodahl, S. Mahmoodian, and S. Stobbe, Rev. Mod. Phys. 87, 347 (2015).

[12] J. A. Mlynek, A. A. Abdumalikov, C. Eichler, and A. Wallraff, Nat. Commun. 5, 5186 (2014).

[13] B. Casabone, K. Friebe, B. Brandstätter, K. Schüppert, R. Blatt, and T. E. Northup, Phys. Rev. Lett. 114, 023602 (2015).

[14] J. Beugnon, M. Jones, J. Dingjan, B. Darquié, G. Messin, A. Browaeys, and P. Grangier, Nature (London) 440, 779 (2006).

[15] A. Neuzner, M. Körber, O. Morin, S. Ritter, and G. Rempe, Nat. Photonics 10, 303 (2016).

[16] D. L. Moehring, P. Maunz, S. Olmschenk, K. C. Younge, D. N. Matsukevich, L.-M. Duan, and C. Monroe, Nature (London) 449, 68 (2007).

[17] R. Wiegner, J. von Zanthier, and G. S. Agarwal, Phys. Rev. A 84, 023805 (2011).

[18] J. I. Cirac and P. Zoller, Phys. Rev. Lett. 74, 4091 (1995).

[19] F. Schmidt-Kaler, H. Häffner, M. Riebe, S. Gulde, G. P. Lancaster, T. Deuschle, C. Becher, C. F. Roos, J. Eschner, and R. Blatt, Nature (London) 422, 408 (2003).

[20] A. Sørensen and K. Mølmer, Phys. Rev. Lett. 82, 1971 (1999).

[21] C. Cabrillo, J. I. Cirac, P. García-Fernández, and P. Zoller, Phys. Rev. A 59, 1025 (1999).

[22] L. Slodička, G. Hétet, N. Röck, P. Schindler, M. Hennrich, and R. Blatt, Phys. Rev. Lett. 110, 083603 (2013).

[23] H. Häffner, F. Schmidt-Kaler, W. Hänsel, C. F. Roos, T. Körber, M. Chwalla, M. Riebe, J. Benhelm, U. D. Rapol, C. Becher, and R. Blatt, Appl. Phys. B 81, 151 (2005).

[24] C. A. Sackett, D. Kielpinski, B. E. King, C. Langer, V. Meyer, C. J. Myatt, M. Rowe, Q. A. Turchette, W. M. Itano, D. J. Wineland, and C. Monroe, Nature (London) 404, 256 (2000).

[25] See Supplemental Material at http://link.aps.org/ supplemental/10.1103/PhysRevLett.120.193603 for a detailed description of the entanglement generation and characterization, the calibration of the fast tuning of the optical path length between the atoms, the full experimental sequence and a brief summary about emission from separable and entangled states, which includes Ref. [41].

[41] L. Slodička, G. Hétet, N. Röck, S. Gerber, P. Schindler, M. Kumph, M. Hennrich, and R. Blatt, Phys. Rev. A 85, 043401 (2012).

[26] J. Suzuki, C. Miniatura, and K. Nemoto, Phys. Rev. A 81, 062307 (2010).

[27] T. Scholak, F. Mintert, and C. A. Müller, Europhys. Lett. 83, 60006 (2008).

[28] F. Schmidt-Kaler and R. Gerritsma, Europhys. Lett. 99, 53001 (2012).

[29] T. Ruster, H. Kaufmann, M. A. Luda, V. Kaushal, C. T. Schmiegelow, F. Schmidt-Kaler, and U. G. Poschinger, Phys. Rev. X 7, 031050 (2017).

[30] R. G. DeVoe and R. G. Brewer, Phys. Rev. Lett. 76, 2049 (1996).

[31] Z. Ficek and R. Tanaś, Phys. Rep. 372, 369 (2002).

[32] C. Navarrete-Benlloch, I. de Vega, D. Porras, and J. I. Cirac, New J. Phys. 13, 023024 (2011).

[33] D. Barredo, S. de Léséleuc, V. Lienhard, T. Lahaye, and A. Browaeys, Science 354, 1021 (2016).

[34] M. Jakob and J. A. Bergou, Opt. Commun. 283, 827 (2010).

[35] G. Vittorini, D. Hucul, I. V. Inlek, C. Crocker, and C. Monroe, Phys. Rev. A 90, 040302(R) (2014).

[36] G. Kurizki, P. Bertet, Y. Kubo, K. Mølmer, D. Petrosyan, P. Rabl, and J. Schmiedmayer, Proc. Natl. Acad. Sci. U.S.A. 112, 3866 (2015).

[37] R. Wiegner, J. Von Zanthier, and G. S. Agarwal, J. Phys. B 44, 055501 (2011).

[38] V. Giovannetti, S. Lloyd, and L. Maccone, Nat. Photonics 5, 222 (2011).

[39] K. Predehl, G. Grosche, S. M. F. Raupach, S. Droste, O. Terra, J. Alnis, Th. Legero, T. W. Hänsch, Th. Udem, R. Holzwarth, and H. Schnatz, Science 336, 441 (2012).

[40] U. Yurtsever, D. Strekalov, and J. P. Dowling, Eur. Phys. J. D 22, 365 (2003). 